\title{
Adaptação do "PourThru" para avaliação de substratos com diferentes granulometrias ${ }^{(1)}$
}

\author{
FERNANDA LUDWIG ${ }^{(2)^{*}}$, AMARALINA CELOTO GUERRERO(3) $^{(3)}$ GUILHERME AMARAL FERREIRA $^{(4)}$ \\ DIRCEU MAXIMINO FERNANDES(5) ${ }^{(5)}$ ROBERTO LYRA VILLAS BÔAS(5)
}

\begin{abstract}
RESUMO
O monitoramento periódico da solução do substrato permite o controle nutricional de plantas de forma mais rápida e prática. Para que esse monitoramento vire rotina entre os produtores de flores envasadas, é necessário a padronização de métodos não destrutivos e de fácil aplicação. O presente trabalho foi conduzido com o objetivo de adaptar a metodologia do "PourThru" para avaliação de substratos com diferentes granulometrias. Foram realizados dois testes para definir o volume de água aplicado e o tempo entre irrigação e coleta do lixiviado, ambos utilizando substratos com granulometrias de 4-2 mm, 2-1 mm, $<4 \mathrm{~mm},<2 \mathrm{~mm}$ e $<1 \mathrm{~mm}$ e quatro repetições. Para o teste I, utilizou-se o esquema fatorial 5 x 4 (5 granulometrias e 4 volumes de água), com volumes de 50, 75, 100 e $125 \mathrm{~mL}$. Para o teste II, utilizou-se o esquema fatorial 5 x 5 (5 granulometrias e 5 tempos de equilíbrio), com tempos de 30, 60, 90, 120 e 150 minutos. A solução lixiviada foi avaliada quanto à condutividade elétrica e ao pH. A metodologia apresentou limitações quando os substratos apresentaram granulometrias entre 4 e $2 \mathrm{~mm}$ e menores que $1 \mathrm{~mm}$. Quanto maior a granulometria, maior a formação de canais preferenciais com a possibilidade de mistura da água usada como veículo e a solução a ser lixiviada. Quanto menor a granulometria, maior a tendência de adsorção dos nutrientes às partículas. Para o substrato de casca de pinus e vaso com 1,3 L de volume, sugere-se o tempo de estabilização de 60 minutos e a aplicação de $75 \mathrm{~mL}$ de água para deslocamento do lixiviado.
\end{abstract}

Palavras-chave: Gerbera jamesonii, condutividade elétrica $\mathrm{pH}$, lixiviado.

\section{ABSTRACT}

Adaptation of "PourThru" for evaluation of substrates with different granulometries

Periodic monitoring of the substrate solution allows the nutritional control of plants to be done more quickly and conveniently. In order to this monitoring become a routine among the producers of potted flowers, a standardization of non-destructive methods and easy application are required. This study was conducted in order to adapt of the "PourThru" methodology for substrate evaluation with different particle sizes. It was conducted on substrates with different particle sizes. Two tests were performed to define the amount of water to be applied, the time between irrigation and the collect of the leachate. Both tests were done using substrates with grain size 4-2 mm, 2-1 mm $<4 \mathrm{~mm}<2 \mathrm{~mm}<1 \mathrm{~mm}$ and four repetitions. For the test I, we used the factorial $5 \mathrm{x} 4$ ( 5 particle sizes and 4 volumes of water), with volumes of 50, 75, 100 and $125 \mathrm{~mL}$. For test II, we used the $5 \times 5$ factorial (5 granulometry balance and 5 times), with times of $30,60,90,120$ and 150 minutes. The leach solution was evaluated for electrical conductivity and $\mathrm{pH}$. The methodology presented showed constraints when the substrates had particle sizes between 4 and $2 \mathrm{~mm}$, and less than $1 \mathrm{~mm}$. The larger the particle sizes is, the greater is the formation of preferential channels that leads to the possibility of mixing the water used as carrier and the solution to be leached is. The smaller the particle size is, the greater is the tendency of adsorption of the nutrients by the particles. For the pine bark substrate and vase with $1.3 \mathrm{~L}$, it is suggested 60 minutes as settling time and the application of $75 \mathrm{~mL}$ of water for leaching the displacement.

Keywords: Gerbera jamesonii, electrical conductivity, $\mathrm{pH}$, leached

DOI: http://dx.doi.org/10.14295/oh.v23i1.911

(1) Recebido em 14/06/2016 e aceito em 19/10/2016

${ }^{(2)}$ Universidade Estadual do Rio Grande do Sul (UERGS), Unidade em Santa Cruz do Sul. Santa Cruz do Sul-RS, Brasil.*Autor correspondente: ludwig.fernanda@yahoo.com.br

${ }^{(3)}$ Universidade Federal de Campina Grande (UFCG), Centro de Ciências e Tecnologia Alimentar, Pombal-PB, Brasil.

${ }^{\left({ }^{4}\right)}$ Centro de Citricultura Sylvio Moreira - APTA. Cordeirópolis-SP, Brasil.

${ }^{(5)}$ Universidade Estadual Paulista “Júlio de Mesquita Filho" (UNESP). Departamento de Recursos Naturais/Ciência do Solo (FCA/

UNESP). Botucatu-SP, Brasil. 


\section{INTRODUÇÃO}

O sistema de produção em substratos vem sendo empregado na maioria dos cultivos comerciais de plantas ornamentais (LUDWIG et al., 2014), e em função dos recipientes apresentarem um volume reduzido para o crescimento radicular, deve-se atentar para a questão nutricional. Para evitar perdas de qualidade, é importante realizar o manejo nutricional, sendo a condutividade elétrica da solução do substrato uma medida indireta de nutrientes disponíveis para as plantas (MOTA et al., 2016; TORRES et al., 2010), que pode ser efetuada de forma rápida no próprio ambiente de cultivo.

Testar a solução do substrato é uma forma precisa para monitorar a verdadeira nutrição do rizosfera, ajudando assim na adaptação do programa de fertilização (YAO et al., 2008). Porém, muitos produtores ainda não realizam a análise periódica devido ao desconhecimento de metodologias apropriadas. Métodos práticos e que se utilizam do substrato na condição de vaso, como o "PourThru", são alternativas para o manejo da concentração de sais (MOTA et al., 2011). Este método se destaca pela natureza não destrutiva e simplicidade na coleta da solução (CAVINS et al., 2004), pois requer somente a adição de água na superfície do substrato e a coleta do lixiviado (YEAGER et al., 1983).

O método do "PourThru" é baseado no deslocamento de um volume de solução, adicionado na parte superior do substrato, com o objetivo de obter amostras de nutrientes através da solução lixiviada (CAVINS, 2002; CAVINS et al., 2008). O procedimento do "PourThru" descrito por Wright (1986) indica que é necessário manter o substrato proximo a capacidade máxima de retenção de água e adicionar uma quantidade suficiente de água destilada de modo que possa ser lixiviado $50 \mathrm{~mL}$ da solução do substrato, a qual é então analisada.

$\mathrm{O}$ "PourThru" é um método rápido e fácil, em que não ocorre distúrbio do sistema radicular durante a extração, o que o torna uma ferramenta de pesquisa viável quando amostras repetidas de um mesmo substrato são necessárias
(YEAGER et al., 1983). A metodologia vem sendo adotada por produtores de flores, porém sem o devido conhecimento das suas particularidades (MOTA et al., 2011), necessitando de maiores recomendações para seu uso na floricultura.

Desse modo, o presente trabalho foi conduzido com o objetivo de adaptar a metodologia do "PourThru" para avaliação de substratos com diferentes granulometrias.

\section{MATERIAL E MÉTODOS}

Os testes foram conduzidos em casa de vegetação com cobertura plástica transparente de $150 \mu \mathrm{m}$ de espessura, laterais de tela branca, cortinas de plástico transparente e pavimento de concreto, em uma área total de $168 \mathrm{~m}^{2}(7,0 \mathrm{x}$ $24,0 \mathrm{~m})$ e $2,6 \mathrm{~m}$ de pé direito.

O delineamento experimental adotado para o teste I foi o de blocos casualizados, empregando o esquema fatorial 5 x 4 (5 granulometrias do substrato e 4 volumes de água para deslocamento da solução) e 4 repetições. Os volumes de água foram de 50, 75, 100 e $125 \mathrm{~mL}$.

O delineamento experimental adotado para o teste II foi o de blocos casualizados, empregando o esquema fatorial 5 x 5 (5 granulometrias do substrato e 5 tempos de equilíbrio) e 4 repetições. Os tempos foram de 30, 60, 90, 120 e 150 minutos.

O substrato utilizado foi composto de $100 \%$ de casca de pinus, o qual foi separado em peneiras afim de obter as seguintes granulometrias: 4 a $2 \mathrm{~mm}$ (material que passou na peneira de $4 \mathrm{~mm}$ e foi retido na de $2 \mathrm{~mm}$ ); 2 a $1 \mathrm{~mm}$ (material que passou na peneira de $2 \mathrm{~mm}$ e foi retida na peneira de $1 \mathrm{~mm}$ ); $<4 \mathrm{~mm}$ (material que passou na peneira de $4 \mathrm{~mm}$ ) $;<2 \mathrm{~mm}$ (material que passou na peneira de $2 \mathrm{~mm}$ ) e $<1 \mathrm{~mm}$ (material que passou na peneira de 1 $\mathrm{mm}$ ). Os materiais foram caracterizados fisicamente para determinar a densidade úmida e seca (HOFFMANN, 1970, citado por BRASIL, 2007) e a curva de retenção de água (DE BOODT; VERDONCK, 1972) e quimicamente para determinar o $\mathrm{pH}$ e condutividade elétrica (BRASIL, 2007). Os resultados das análises para os substratos são apresentados na Tabela 1. 
Tabela 1. Características químicas e físicas dos substratos.

Table 1. Chemical and physical characteristics of substrates.

\begin{tabular}{|c|c|c|c|c|c|}
\hline \multirow{2}{*}{$\begin{array}{l}\text { Características } \\
\text { Characteristics }\end{array}$} & \multicolumn{5}{|c|}{$\begin{array}{l}\text { Substratos } \\
\text { Substrates }\end{array}$} \\
\hline & 1 & 2 & 3 & 4 & 5 \\
\hline $\begin{array}{l}\text { CE } 1: 5\left(\mathrm{dS} \mathrm{m} \mathrm{m}^{-1}\right) \\
\text { EC } 1: 5\left(d S m^{-1}\right)\end{array}$ & 0,29 & 0,15 & 0,34 & 0,38 & 0,29 \\
\hline $\begin{array}{l}\mathrm{pH} 1: 5 \\
\mathrm{pH} 1: 5\end{array}$ & 3,47 & 3,49 & 4,17 & 3,49 & 3,47 \\
\hline $\begin{array}{l}\text { Porosidade total (\%) } \\
\text { Total porosity (\%) }\end{array}$ & 82,37 & 80,94 & 83,08 & 80,62 & 79,78 \\
\hline $\begin{array}{l}\text { Sólidos (\%) } \\
\text { Solid (\%) }\end{array}$ & 17,63 & 19,06 & 16,92 & 19,38 & 20,22 \\
\hline $\begin{array}{l}\text { Aeração (\%) } \\
\text { Aeration (\%) }\end{array}$ & 41,55 & 35,04 & 25,65 & 16,80 & 7,91 \\
\hline $\begin{array}{c}\text { Água Facilmente Disponível (\%) } \\
\text { Easily available water }\end{array}$ & 14,76 & 18,67 & 28,15 & 33,92 & 34,29 \\
\hline $\begin{array}{l}\text { Água Tamponante (\%) } \\
\text { Buffering water (\%) }\end{array}$ & 1,53 & 2,55 & 3,27 & 5,77 & 15,31 \\
\hline $\begin{array}{l}\text { Água Disponível (\%) } \\
\text { Water available (\%) }\end{array}$ & 16,29 & 21,22 & 31,42 & 39,69 & 49,60 \\
\hline $\begin{array}{l}\text { Água Remanescente (\%) } \\
\text { Water remainder (\%) }\end{array}$ & 24,53 & 24,69 & 26,00 & 24,12 & 22,27 \\
\hline $\begin{array}{l}\text { Densidade úmida }\left(\mathrm{kg} \mathrm{m}^{-3}\right) \\
\text { Moist bulk density }\left(\mathrm{kg} \mathrm{m}^{-3}\right)\end{array}$ & 391,18 & 526,00 & 490,55 & 463,76 & 548,04 \\
\hline $\begin{array}{l}\text { Densidade seca }\left(\mathrm{kg} \mathrm{m}^{-3}\right) \\
\text { Dry bulk density }\left(\mathrm{kg} \mathrm{m}^{-3}\right)\end{array}$ & 216,38 & 312,61 & 239,75 & 268,38 & 280,75 \\
\hline
\end{tabular}

Substratos (substrates): $1=4-2 \mathrm{~mm} ; 2=2-1 \mathrm{~mm} ; 3=<4 \mathrm{~mm} ; 4=<2 \mathrm{~mm} ; 5=<1 \mathrm{~mm}$.

Foram utilizadas plantas de Gerbera jamesonii, para cultivo em vaso, cultivar Cherry, pertencente ao grupo Festival e série "Dark Eyes". Estas foram conduzidas em vaso plástico com 1,3 L de volume e dimensões de 12,2 $\mathrm{cm}$ de altura, $14,8 \mathrm{~cm}$ de base superior e $9,8 \mathrm{~cm}$ de base inferior, preenchidos com o substrato correspondente ao tratamento, de acordo com a densidade úmida.

Os testes foram realizados em vasos contendo plantas de gérbera que foram conduzidas com a solução nutritiva adaptada de Ludwig et al. (2008), a qual apresentou a

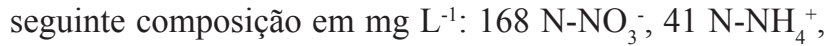
$303 \mathrm{~K}, 35 \mathrm{P}, 105 \mathrm{Ca}, 45 \mathrm{Mg}$ e $55 \mathrm{~S}, 0,19 \mathrm{~B}, 0,08 \mathrm{Cu}, 2,74$ $\mathrm{Fe}, 0,19 \mathrm{Mn}, 0,04 \mathrm{Mo}$ e 0,08 Zn. A condutividade elétrica (CE) da solução apresentou valores de $2,5 \mathrm{dS} \mathrm{m}^{-1} \pm 30 \%$.

As análises da $\mathrm{CE}\left(\mathrm{dS} \mathrm{m}^{-1}\right)$ e do $\mathrm{pH}$ da solução lixiviada do substrato foram realizadas quando as plantas estavam em ponto de comercialização, utilizando a metodologia do "PourThru" (CAVINS et al., 2000), com as adaptações relativas aos tratamentos.

Para o teste I, um vaso por parcela foi saturado com a solução nutritiva, e depois de transcorrido 60 minutos foi adicionado o volume de água correspondente aos tratamentos $(50,75,100$ e $125 \mathrm{~mL})$, de modo que a solução do substrato fosse lixiviada para o interior de sacos plásticos colocados na parte inferior dos vasos. Para o teste II, um vaso por parcela foi saturado com a solução nutritiva, e depois de transcorrido o tempo de cada tratamento $(30,60,90,120$ e 150 minutos $)$ foi adicionado $75 \mathrm{~mL}$ de água, para promover o deslocamento da solução para o interior dos sacos plásticos.

As análises de $\mathrm{CE}$ e pH foram realizadas na solução lixiviada, imediatamente após a coleta. A CE foi determinada em condutivímetro de bancada da marca Digimed DM-3 e o pH com potenciômetro de bancada da marca Digimed DM-2.

Os resultados foram submetidos à análise de variância. Os efeitos das granulometrias tiveram suas médias comparadas pelo teste Tukey a 5\% de probabilidade, quando significativos. Os efeitos dos volumes de água e o tempo de equilíbrio foram submetidos à análise de regressão, quando significativos, testando os modelos lineares e quadráticos, adotando-se a equação de regressão que melhor se ajustava aos dados, escolhida com base na significância dos coeficientes de regressão a $1 \%(* *)$ e $5 \%$ (*) de probabilidade pelo teste $\mathrm{F}$ e no maior valor do coeficiente de determinação $\left(\mathrm{R}^{2}\right)$, com o uso do programa estatístico Sisvar (FERREIRA, 2011).

\section{RESULTADOS E DISCUSSÃO}

A análise de variância revelou efeito significativo para os valores de condutividade elétrica (CE) em função das granulometrias dos substratos (Tabela 2). Não houve efeito da solução nutritiva e da interação entre a granulometria e a solução nutritiva. 
Tabela 2. Valor médio de condutividade elétrica da solução do substrato em função da granulometria do substrato e volume de água.

Table 2. Average value of electrical conductivity of substrate solution depending on the particle size of the substrate and the volume of water.

\begin{tabular}{|c|c|c|c|c|c|}
\hline \multirow{4}{*}{$\begin{array}{l}\text { Granulometria } \\
\text { Granulometry }\end{array}$} & \multicolumn{5}{|c|}{$\begin{array}{l}\text { CE } \\
E C\end{array}$} \\
\hline & \multicolumn{5}{|c|}{$\begin{array}{l}\text { Volume }(\mathrm{mL}) \\
\text { Volume }(m L)\end{array}$} \\
\hline & 50 & 75 & 100 & 125 & $\begin{array}{l}\text { Média } \\
\text { Average }\end{array}$ \\
\hline & \multicolumn{5}{|c|}{ 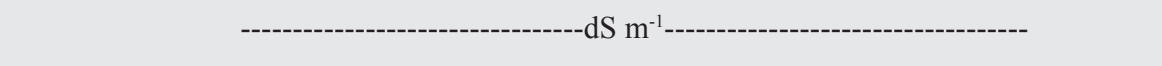 } \\
\hline $4-2 \mathrm{~mm}$ & 1,67 & 1,46 & 2,11 & 1,24 & $1,62 \mathrm{~b}$ \\
\hline $2-1 \mathrm{~mm}$ & 2,01 & 1,84 & 2,60 & 2,91 & $2,34 \mathrm{~b}$ \\
\hline$<4 \mathrm{~mm}$ & 5,32 & 2,69 & 4,01 & 3,37 & $3,85 \mathrm{a}$ \\
\hline$<2 \mathrm{~mm}$ & 2,98 & 3,31 & 3,83 & 4,09 & $3,55 \mathrm{a}$ \\
\hline$<1 \mathrm{~mm}$ & - & 3,03 & 3,28 & 4,30 & $3,54 \mathrm{a}$ \\
\hline G & \multicolumn{5}{|c|}{$* *$} \\
\hline V & \multicolumn{5}{|c|}{ NS } \\
\hline $\mathrm{G}^{*} \mathrm{~V}$ & \multicolumn{5}{|c|}{ NS } \\
\hline CV (\%) & \multicolumn{5}{|c|}{31,28} \\
\hline
\end{tabular}

Médias seguidas pela mesma letra na coluna não diferem significativamente pelo teste Tukey a 1 e 5\%. G: granulometria; V: volume. Means followed by the same letter in the column do not differ significantly by Tukey test at $5 \%$. G: granulometry; V: volume.

Os menores valores de CE foram obtidos nos substratos com maiores granulometrias (Tabela 2). Partículas de tamanhos maiores promovem menor retenção de água e maior espaço de aeração ao substrato, o qual pode não se manter na capacidade máxima de retenção de água após transcorrido uma hora da saturação. Nos substratos com maior espaço de aeração, a água usada como veículo pode mover-se rapidamente através de canais criados pelos macroporos e misturar-se à solução, diluindo a concentração de sais com consequente redução dos valores da CE.

Para o substrato com granulometrias entre 4 e $2 \mathrm{~mm}$, independentemente do volume de água aplicada, os valores de CE do lixiviado foram inferiores ao fornecido, o qual era mantido na faixa de $2,5 \mathrm{dSm}^{-1} \pm 30 \%$. Esse substrato apresentou o maior espaço de aeração, de 41,55\% (Tabela 1), sugerindo a possível mistura da água com a solução. $\mathrm{O}$ resultado indica que o método não é adequado para substratos com granulometrias que confiram espaço de aeração superior a $40 \%$.

Para o substrato com granulometria $<1 \mathrm{~mm}$, o volume de $50 \mathrm{~mL}$ de água não foi suficiente para promover o deslocamento da solução. Esse substrato apresenta a característica de reduzido espaço de aeração e elevada retenção de água (Tabela 1), mantendo a água fortemente aderida aos microporos. Para essa granulometria, os valores de $\mathrm{CE}$ que se enquadraram na referência foram aqueles obtidos com o volume de $75 \mathrm{~mL}$.

O recomendado é que pelo menos $50 \mathrm{~mL}$ de solução seja lixiviada para que possa ser realizada a leitura (CAVINS et al., 2000). Esse volume irá fornecer aos produtores informações consistentes para que os resultados possam ser comparados com os valores referenciais (TORRES et al., 2010). Em função da menor quantidade de solução lixiviada quando o volume de água foi de 50 $\mathrm{mL}$, recomenda-se que seja aplicado pelo menos $75 \mathrm{~mL}$ para deslocamento da solução do substrato. Também, como esse volume possibilitou leituras de CE mais próximas aos valores referenciais, sugere-se sua aplicação como padrão, em plantas de gérbera conduzidas em vaso de $12,2 \mathrm{~cm}$ de altura.

Para plantas de Phalaenopsis conduzidas em vasos de $10,5 \mathrm{~cm}$ de altura e preenchidos com substrato a base de esfagno, Yao et al. (2008) verificaram que volumes de água entre 40 a $70 \mathrm{~mL}$ não alteraram os valores de $\mathrm{CE}$ e $\mathrm{pH}$ da solução lixiviada, recomendando a aplicação de $60 \mathrm{~mL}$, para deslocamento de $50 \mathrm{~mL}$ de solução. Wright (1986) também constatou que volumes entre 40 e $100 \mathrm{~mL}$ não influenciam na concentração dos nutrientes extraídos, em recipientes de 3,8 litros. 
Para os substratos com granulometrias de 2 a $1 \mathrm{~mm} \mathrm{e}<1$ $\mathrm{mm}$, foi possível ajustar uma equação linear decrescente em função do tempo de estabilização, para o valor de CE (Figura 1). Ao longo do tempo pode ter ocorrido uma adsorção dos nutrientes às partículas ou a absorção destes pelas plantas. Cavins et al. (2005) observaram que substratos com maior retenção de umidade apresentaram redução de sais na solução lixiviada, devido ao maior acumulo desses no substrato.

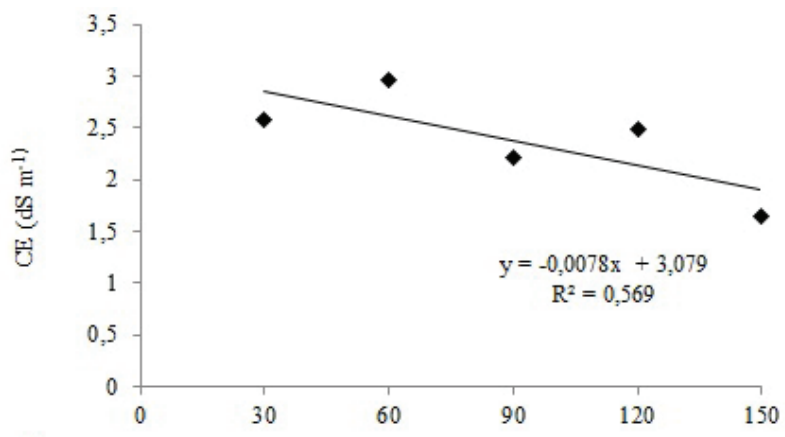

A

Tempo (min)

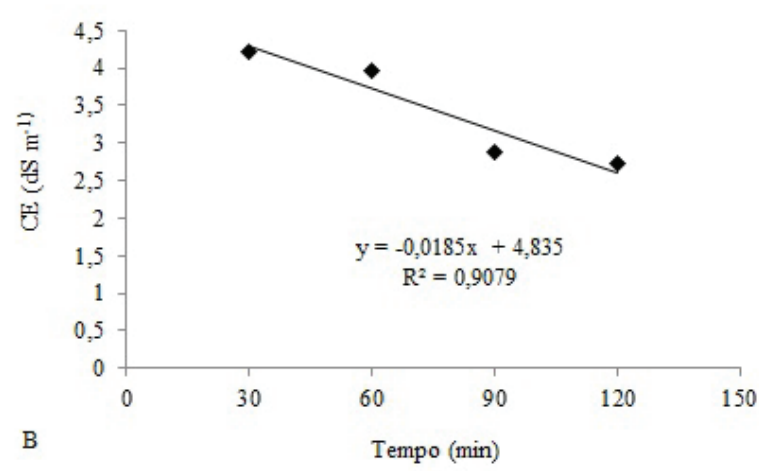

Figura 1. Valores médios de condutividade elétrica da solução dos substratos com granulometria de 2-1 mm (A) e < 4 mm (B), em função do tempo de estabilização.

Figure 1. Mean values of electrical conductivity of the substrate solution with a particle size of 2-1 $\mathrm{mm}(A)$ and $<4 \mathrm{~mm}(B)$, due to the settling time.

Para os substratos com menores granulometrias, não foi possível extrair a solução para aferição dos valores, no tempo de 150 minutos (Tabela 3). Como a retenção de água nesses substratos era maior (Tabela 1), ao longo do tempo a solução nutritiva tornou-se indisponível, devido a retenção nos microporos existentes em maior quantidade.

Tabela 3. Valor médio de condutividade elétrica da solução do substrato em função da granulometria do substrato e tempo de estabilização.

Table 3. Average value of electrical conductivity of the substrate solution depending on the particle size of the substrate and settling time.

\begin{tabular}{|c|c|c|c|c|c|c|}
\hline \multirow{4}{*}{$\begin{array}{l}\text { Granulometria } \\
\text { Granulometry }\end{array}$} & \multicolumn{6}{|c|}{$\begin{array}{l}\text { CE } \\
E C\end{array}$} \\
\hline & \multicolumn{6}{|c|}{$\begin{array}{c}\text { Tempo (min) } \\
\text { Time (min) }\end{array}$} \\
\hline & 30 & 60 & 90 & 120 & 150 & $\mathrm{R}$ \\
\hline & \multicolumn{5}{|c|}{ 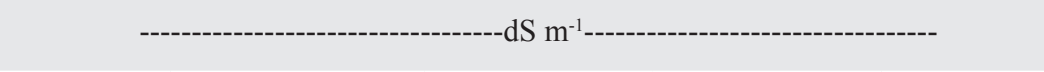 } & \\
\hline $4-2 \mathrm{~mm}$ & $1,21 \mathrm{c}$ & $1,33 \mathrm{c}$ & $1,36 \mathrm{c}$ & $1,21 \mathrm{a}$ & $1,05 \mathrm{~b}$ & NS \\
\hline $2-1 \mathrm{~mm}$ & $2,57 \mathrm{bc}$ & $2,97 \mathrm{ab}$ & $2,21 \mathrm{bc}$ & $2,48 \mathrm{a}$ & $1,64 \mathrm{~b}$ & $\mathrm{~L}^{*}$ \\
\hline$<4 \mathrm{~mm}$ & $2,09 \mathrm{bc}$ & $2,66 a b c$ & $2,37 \mathrm{ab}$ & $1,27 \mathrm{a}$ & $2,93 \mathrm{a}$ & $\mathrm{L}^{* *}$ \\
\hline$<2 \mathrm{~mm}$ & $3,42 \mathrm{ab}$ & $2,55 \mathrm{bc}$ & $4,66 \mathrm{a}$ & $2,56 \mathrm{a}$ & - & NS \\
\hline$<1 \mathrm{~mm}$ & $4,22 \mathrm{a}$ & $3,96 \mathrm{a}$ & $2,88 \mathrm{~b}$ & $2,73 \mathrm{a}$ & - & NS \\
\hline G & \multicolumn{5}{|c|}{$* *$} & \\
\hline $\mathrm{T}$ & \multicolumn{5}{|c|}{$* *$} & \\
\hline $\mathrm{G}^{*} \mathrm{~T}$ & \multicolumn{5}{|c|}{$*$} & \\
\hline CV (\%) & \multicolumn{5}{|c|}{24,64} & \\
\hline
\end{tabular}

Médias seguidas pela mesma letra na coluna não diferem significativamente pelo teste Tukey a 1 e 5\%. G: granulometria; V: volume. R: regressão. Means followed by the same letter in the column do not differ significantly by Tukey test at $5 \%$. G: granulometry; V: volume. R: regression. 
Para os substratos com granulometria de 2-1 $\mathrm{mm} \mathrm{e}$ $<4 \mathrm{~mm}$, o tempo de 30 minutos foi suficiente para que os valores de $\mathrm{CE}$ ficassem próximos à referência. Para os demais substratos, foram necessários pelo menos 60 minutos. Torres et al. (2010) sugerem que a cultura seja irrigada de 30 a 60 minutos antes da coleta do lixiviado para garantir que o substrato esteja na capacidade máxima de retenção de água.

Segundo Yao et al. (2008), 20 minutos é o tempo mínimo necessário para o equilíbrio da solução. Porém, Cavins et al. (2005) sugerem o tempo de 60 minutos para permitir o equilíbrio de nutrientes e obter uma amostra representativa do estado nutricional disponível para a planta. Segundo os mesmos autores, após 120 minutos podem ocorrer mudanças rápidas no teor de água nos recipientes e diminuir o volume lixiviado. Wright (1986) recomenda que o "PourThru" seja conduzido quando o nível de umidade do substrato esteja próximo à capacidade de recipiente ou máxima retenção de água, e mais importante que o nível de umidade deve ser aproximadamente o mesmo em todas as extrações. As alterações de umidade podem ocorrer devido a transpiração ativa das plantas, a qual varia em função da temperatura e umidade relativa do ar no ambiente de cultivo.

Compton e Nelson (1997) sugerem que a solução seja deslocada entre 1 a 2 horas após a fertirrigação, para plantas conduzidas em bandejas de 288 células. Segundo os autores, um período menor que 1 hora entre a fertirrigação e a coleta pode ser inadequado para o equilíbrio dos nutrientes na solução, porém períodos maiores poderiam refletir os níveis de nutrientes já absorvidos pelas plantas.

Os valores de $\mathrm{pH}$ variaram somente em função da granulometria do substrato, não sendo influenciados pelo tempo de estabilização (Tabela 4). Resultados semelhantes quanto ao tempo de estabilização foram obtidos por Cavins et al. (2005), os quais sugerem que o efeito tenha ocorrido em função do elevado volume do substrato $(1,3$ L a 2,0 L), o que aumenta a capacidade de tamponamento do sistema e a alteração da solução do substrato até 240 minutos da irrigação.

Tabela 4. Valor médio de $\mathrm{pH}$ da solução do substrato em função da granulometria do substrato e volume de água.

Table 4. Average value of $\mathrm{pH}$ of the substrate solution depending on the particle size of the substrate and the volume of water.

\begin{tabular}{|c|c|c|c|c|c|}
\hline \multirow{3}{*}{$\begin{array}{c}\text { Granulometria } \\
\text { Granulometry }\end{array}$} & \multicolumn{5}{|c|}{ pH } \\
\hline & \multicolumn{5}{|c|}{$\begin{array}{l}\text { Volume }(\mathrm{mL}) \\
\text { Volume }(m L)\end{array}$} \\
\hline & 50 & 75 & 100 & 125 & $\begin{array}{l}\text { Média } \\
\text { Average }\end{array}$ \\
\hline $4-2 \mathrm{~mm}$ & 5,99 & 6,14 & 5,94 & 5,94 & $6,00 \mathrm{a}$ \\
\hline $2-1 \mathrm{~mm}$ & 5,93 & 5,86 & 5,82 & 5,78 & $5,85 \mathrm{ab}$ \\
\hline$<4 \mathrm{~mm}$ & 5,69 & 5,89 & 5,81 & 5,68 & $5,77 \mathrm{~b}$ \\
\hline$<2 \mathrm{~mm}$ & 5,95 & 5,86 & 5,90 & 5,71 & $5,85 \mathrm{ab}$ \\
\hline$<1 \mathrm{~mm}$ & - & 5,88 & 5,77 & 5,74 & $5,80 \mathrm{~b}$ \\
\hline G & \multicolumn{5}{|c|}{$* *$} \\
\hline V & \multicolumn{5}{|c|}{$\mathrm{L}^{*}$} \\
\hline $\mathrm{G} * \mathrm{~V}$ & \multicolumn{5}{|c|}{ NS } \\
\hline CV (\%) & \multicolumn{5}{|c|}{2,47} \\
\hline
\end{tabular}

Médias seguidas pela mesma letra na coluna não diferem significativamente pelo teste Tukey a 1 e $5 \%$. G: granulometria; V: volume. Means followed by the same letter in the column do not differ significantly by Tukey test at $5 \%$. G: granulometry; V: volume.w

Valores superiores de $\mathrm{pH}$ foram obtidos no substrato com granulometria de 4-2 mm e inferiores nos substratos com granulometrias menores que $2 \mathrm{~mm}$ (Tabela 5). Quando o tempo de estabilização foi de 150 minutos, não foi possível coletar volume de solução suficiente para proceder a leitura. Desse modo, para avaliação do $\mathrm{pH}$, os tempos entre 30 e 120 minutos podem ser utilizados, sem que haja alteração significativa na leitura. 
Tabela 5. Valor médio de pH da solução do substrato em função da granulometria do substrato e tempo de estabilização. Table 5. Average value of $\mathrm{pH}$ of the substrate solution depending on the particle size of the substrate and settling time

\begin{tabular}{|c|c|c|c|c|c|c|}
\hline \multirow{3}{*}{$\begin{array}{c}\text { Granulometria } \\
\text { Granulometry }\end{array}$} & \multicolumn{6}{|c|}{ pH } \\
\hline & \multicolumn{6}{|c|}{$\begin{array}{c}\text { Tempo (min) } \\
\text { Time (min) }\end{array}$} \\
\hline & 30 & 60 & 90 & 120 & 150 & $\begin{array}{l}\text { Média } \\
\text { Average }\end{array}$ \\
\hline 4-2 mm & 6,11 & 5,98 & 6,23 & 6,04 & 6,08 & $6,08 \mathrm{a}$ \\
\hline $2-1 \mathrm{~mm}$ & 5,84 & 5,81 & 5,99 & 5,94 & 5,98 & $5,91 \mathrm{~b}$ \\
\hline$<4 \mathrm{~mm}$ & 5,88 & 5,84 & 5,94 & 6,00 & 5,77 & $5,89 \mathrm{~b}$ \\
\hline$<2 \mathrm{~mm}$ & 5,77 & 5,81 & 5,72 & 5,78 & - & $5,77 \mathrm{c}$ \\
\hline$<1 \mathrm{~mm}$ & 5,84 & 5,89 & 5,85 & 5,93 & - & $5,87 \mathrm{bc}$ \\
\hline $\mathrm{S}$ & \multicolumn{6}{|c|}{$* *$} \\
\hline $\mathrm{T}$ & \multicolumn{6}{|c|}{ NS } \\
\hline $\mathrm{S} * \mathrm{~T}$ & \multicolumn{6}{|c|}{ NS } \\
\hline CV (\%) & \multicolumn{6}{|c|}{1,65} \\
\hline
\end{tabular}

Médias seguidas pela mesma letra na coluna não diferem significativamente pelo teste Tukey a 5\%. G: granulometria; V: volume. Means followed by the same letter in the column do not differ significantly by Tukey test at $5 \%$. G: granulometry; V: volume.

Os valores médios de $\mathrm{pH}$ da solução do substrato variaram significativamente em função da granulometria e do volume de água aplicado (Tabela 4). Os maiores valores de $\mathrm{pH}$ foram obtidos nos substratos com granulometrias de 4 a $2 \mathrm{~mm}$, os quais não variaram significativamente nas granulometrias 2 a $1 \mathrm{~mm} \mathrm{e}<2 \mathrm{~mm}$ (Tabela 4 ).

Quanto maior o volume de água aplicado para coleta do lixiviado, os valores de $\mathrm{pH}$ reduziram (Figura 2). Torres et al. (2010), avaliando diferentes volumes de recipiente e volumes de lixiviado, também verificaram que o $\mathrm{pH}$ reduziu em função do aumento da água aplicada para deslocamento do lixiviado. Os autores sugerem que seja utilizado o critério de coleta de $50 \mathrm{~mL}$ de lixiviado para análise do $\mathrm{pH}$, independente do volume do recipiente. Yeager et al. (1983), também observaram redução significativa nos valores de $\mathrm{pH}$, à medida que a água era aumentada até $100 \mathrm{~mL}$, em vasos com volume de três litros. Já Yao et al. (2008) constataram que a variação do pH ocorreu em função do ciclo de cultivo e não em função do volume de deslocamento de 30 a $70 \mathrm{~mL}$.

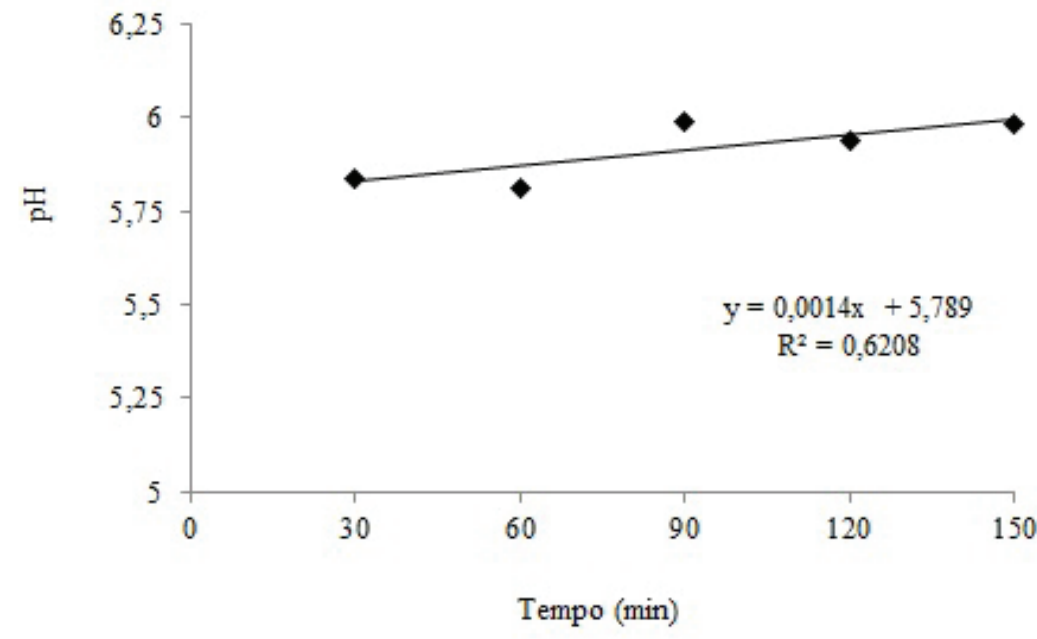

Figura 2. Valores médios de $\mathrm{pH}$ da solução do substrato em função do volume.

Figure 2. Average $p H$ values of substrate solution by volume. 


\section{CONCLUSÕES}

A metodologia apresentou limitações quando os substratos apresentaram granulometrias entre 4 e $2 \mathrm{~mm}$ e menores que $1 \mathrm{~mm}$. Quanto maior a granulometria, maior a formação de canais preferenciais com a possibilidade de mistura da água usada como veículo e a solução a ser lixiviada. Quanto menor a granulometria, maior a tendência de adsorção dos nutrientes às partículas.

Para o substrato de casca de pinus e vaso com 1,3 L de volume, sugere-se o tempo de estabilização de 60 minutos e a aplicação de $75 \mathrm{~mL}$ de água para deslocamento do lixiviado.

\section{REFERÊNCIAS}

BRASIL. Instrução Normativa n.17, de 21 de maio de 2007. Aprova os Métodos Analíticos Oficiais para Análise de Substratos e Condicionadores de Solos. Diário Oficial da União, Brasília, 24 maio. 2007. Seção 1, p.8.

CAVINS, T.J. Adaptation of the pourthru nutrient extraction procedures to greenhouse crop production. Raleigh: Faculty of North Caroline State University, 2002. 148p.

CAVINS, T.J.; WHIPKER, B.E.; FONTENO, W.C. Establishment of calibration curves for comparing pourthrough and saturated media extract nutrient values. HortScience, v.39, p.1635-1639, 2004.

CAVINS, T.J.; WHIPKER, B.E.; FONTENO, W.C. Timing of PourThru affects $\mathrm{pH}$, electrical conductivity and leachate volume. Communications in Soil Science and Plant Analysis, v.36, p.1573-1581, 2005.

CAVINS, T.J.; WHIPKER, B.E.; FONTENO, W.C.; HARDEN, B.; McCALL, I.; GIBSON, J.L. Monitoring and managing $\mathrm{pH}$ and $\mathrm{EC}$ using the PourThru extraction method. Raleigh: Horticulture Information, 2000. $17 \mathrm{p}$.

COMPTON, A.J; NELSON, P.V. Timing is Crucial for Plug Seedling Substrate Testing. HortTechnology, v.7, p.63-68, 1997.

De BOODT, M.; VERDONCK, O. The physical properties of the substrates in horticulture. Acta Horticulturae, v.26, p. 37-44, 1972.
FERREIRA, D.F. Sisvar: A computer statistical analysis system. Ciência e Agrotecnologia, v.35, p.1039-1042, 2011. DOI: <http://dx.doi.org/10.1590/10.1590/S141370542011000600001>

LUDWIG, F.; FERNANDES, D.M.; GUERRERO, A.C.; VILLAS BÔAS, R.L. Características dos substratos na absorção de nutrientes e na produção de gérbera de vaso. Horticultura Brasileira, v.32, p.184-189, 2014. DOI: $<\mathrm{http}$ // dx.doi.org/10.1590/S0102-05362014000200011>

LUDWIG, F.; FERNANDES, D.M.; MOTA, P.R.D.; VILLAS BÔAS, R.L. Macronutrientes em cultivares de gérbera sob dois níveis de fertirrigação. Horticultura Brasileira, v.26, p.68-73, 2008. DOI: <http://dx.doi. org/10.1590/ S0102-05362008000100013>

MOTA, P.R.D.; VILLAS BÔAS, R.L.; LUDWIG, F.; FERNANDES, D.M. Development and mineral nutrition of gerbera planta as a function of electrical conductivity. Ornamental Horticulture, v.22, n.1, p.37-42. 2016. DOI: <http://dx.doi.org/10.14295/aohl.v21i2.533>

MOTA, P.R.D.; VILLAS BÔAS, R.L.; LUDWIG, F.; FERNANDES, D.M.; FOLEGATTI, M.V. Avaliação do $\mathrm{pH}$ e da condutividade elétrica do substrato sob níveis de fertirrigação e métodos de extração da solução. Revista Brasileira de Horticultura Ornamental, v.17, p.121-126, 2011. DOI: <http://dx.doi.org/10.14295/rbho.v17i2.707>

TORRES, A.P.; MICKELBART, M.V.; LOPEZ, R.G. Leachate volume effects on $\mathrm{pH}$ and electrical conductivity measurements in containers obtained using the Pourthrough method. HortTechnology, v.20, p. 608-611, 2010.

WRIGHT, R.D. The Pour-through nutrient extraction procedure. HortScience, v.21, p.227-229, 1986.

YAO, H.Y.; R.S.; HO, S.B. CHANG, Y.C.A. Adapting the Pour-through medium extraction method to Phalaenopsis grown in sphagnum moss. Hortscience, v.43, p.21672170, 2008

YEAGER, T.H.; WRIGHT, R.D.; DONOHUE, S.J. Comparison of Pour-through and satured pine bark extract $\mathrm{N}, \mathrm{P}, \mathrm{K}$ and $\mathrm{pH}$ levels. Journal of American Society for Horticultural Science, v.108, p.112-114. 1983. 